\title{
Control of the Radiation of a Silicon-Based Optical Leaky Wave Antenna through Optical Pumping
}

\author{
Salvatore Campione, Qi Song, Caner Guclu, Ozdal Boyraz, and Filippo Capolino \\ Department of Electrical Engineering and Computer Science, University of California Irvine, CA, 92697 USA \\ Author e-mail address: oboyraz@uci.edu, f.capolino@uci.edu
}

\begin{abstract}
We propose the control of the radiation of an optical leaky wave antenna through optical pumping schemes. A bi-directional pumping is eventually adopted, and the tunability of the radiation is observed.
\end{abstract}

\section{Introduction}

Optical antennas have the capabilities to enhance the interaction between light and matter, and thus have the potential to boost the efficiency of optoelectronic devices such as light-emitting diodes, lasers and solar cells, and optical sensors. Recently, we have introduced the concept of an optical leaky wave antenna (OLWA) that provides directive radiation at $1550 \mathrm{~nm}$ through the excitation of a leaky wave (LW) guided mode into a dielectric waveguide comprising periodic silicon perturbations [1,2]. Very directive near-IR optical antennas with controlled beam steering and radiation pattern are the subject of great interest for applications such as planar imaging and LIDAR. Also, the dielectric waveguide used in the proposed antenna facilitate operations from visible to mid-IR and provide unique opportunities for active device control. In this work, we propose the optical control of the radiation by using visible light pumping. We show that co-propagating visible light at $600 \mathrm{~nm}$ can control the radiation direction and directivity of the leaky wave radiation at $1550 \mathrm{~nm}$. All the simulations are done with Ansys HFSS and COMSOL Multiphysics which provide good agreement of the results.

\section{Radiated power tunability via optical pumping}

We focus on the 2D model of the antenna in Fig. 1 (the agreement between 2D and 3D calculations has been shown in $[2])$.

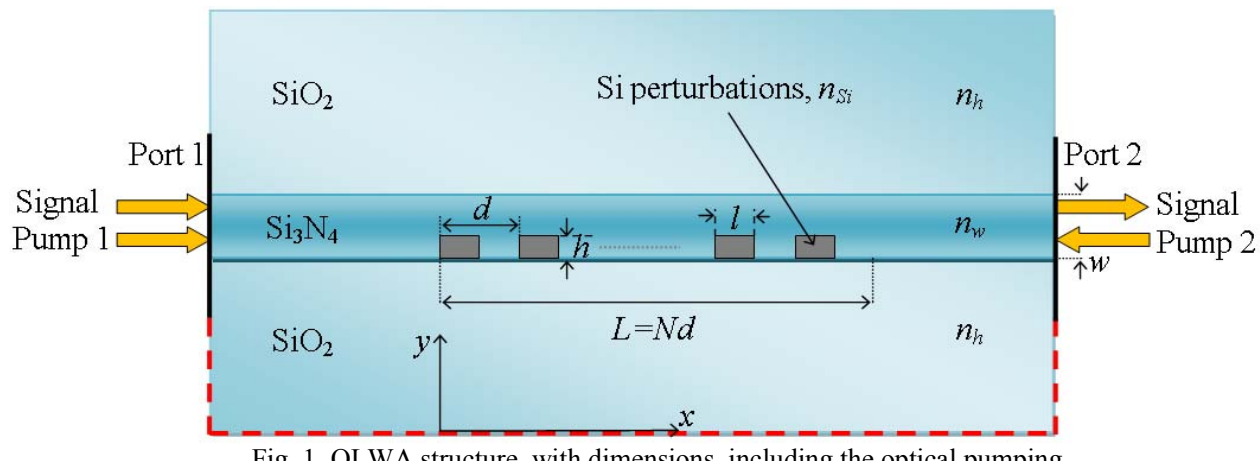

Fig. 1. OLWA structure, with dimensions, including the optical pumping.

The waveguide is made of silicon nitride $\left(\mathrm{Si}_{3} \mathrm{~N}_{4}\right)$ with refractive index $n_{w}=1.67$, is positioned along the $x$ direction and is $100 \mu \mathrm{m}$ long and it has a width $w=1 \mu \mathrm{m}$ along the $y$ direction. The silica glass $\left(\mathrm{SiO}_{2}\right)$ computational domain has a refractive index $n_{h}=1.45$, is $100 \mu \mathrm{m}$ long along the $x$ direction and $20 \mu \mathrm{m}$ wide in the $y$ direction. For simplicity, the structure is invariant along the $z$ direction. To provide direct radiation, we utilize $N=60$ silicon (Si) perturbations, with refractive index $n_{S i}$ (which is a frequency dependent parameter), positioned periodically along the $x$ direction in the bottom side of the waveguide (along $y$ ), and centered with respect to the center of the waveguide (along $x$ ). The period of the perturbations is $d=970 \mathrm{~nm}$ (each element has a length $l=d / 2$ ), and has a height of $h=0.3 \mu \mathrm{m}$ along $y$. A guided wave (Signal) is injected from the left side (port size is $2.4 \mu \mathrm{m}$ ) of the antenna at $1550 \mathrm{~nm}\left(n_{S i}=3.48\right)$, with electric field polarized along $z$. The working principle of the OLWA has been outlined in [2]. Here, we utilize two pump signals at $600 \mathrm{~nm}\left(n_{S i}=3.939+i 0.02\right)$ co-propagating and counterpropagating with the signal to generate electron-hole pairs in the silicon perturbations that can alter both the real $\left(\Delta n_{S i}\right)$ and the imaginary $\left(\Delta k_{S i}\right.$ ) parts of the Si refractive index (unitless) at $1550 \mathrm{~nm}$ as described by the Drude's model [3]

$$
\Delta n_{S i}\left(N_{e}, N_{h}\right)=-\left(8.8 \times 10^{-4} N_{e}+8.5 N_{h}^{0.8}\right) \times 10^{-18}, \quad \Delta k_{S i}\left(N_{e}, N_{h}\right)=\frac{\left(8.5 N_{e}+6.0 N_{h}\right) \times 10^{-16}}{k_{0}},
$$


where $N_{e}$ and $N_{h}$ are the concentrations of electrons and holes (expressed in $\mathrm{cm}^{-3}$ ) in $\mathrm{Si}$, and $k_{0}$ is the free space wavenumber at $1550 \mathrm{~nm}$ (expressed in $\mathrm{m}^{-1}$ ). The concentrations of electrons and holes are computed through the absorbed power in the silicon perturbations $\left(P_{\mathrm{abs}, \mathrm{Si}}\right)$ due to the visible pumping light at $600 \mathrm{~nm}$ as

$$
N_{e}=N_{h}=\frac{P_{\mathrm{abs}, \mathrm{Si}}}{h v} \times \frac{\tau}{V} \times 10^{-6}
$$

where $h v$ is the photon energy at $600 \mathrm{~nm}, \tau$ is the lifetime of the electrons and holes (assumed to be $1 \mathrm{~ns}$ ) and $V$ is the volume of the silicon perturbation. The profile of carrier concentration versus silicon perturbation cell is shown in Fig. 2(a) for single pump co-propagating with signal at $1550 \mathrm{~nm}$, and in Fig. 2(b) for bi-directional pumping configuration, assuming an input power density of $4.17 \times 10^{5} \mathrm{~W} / \mathrm{cm}^{2}$ (assuming a thickness along $z$ of $0.1 \mu \mathrm{m}$ ) associated to each pump.
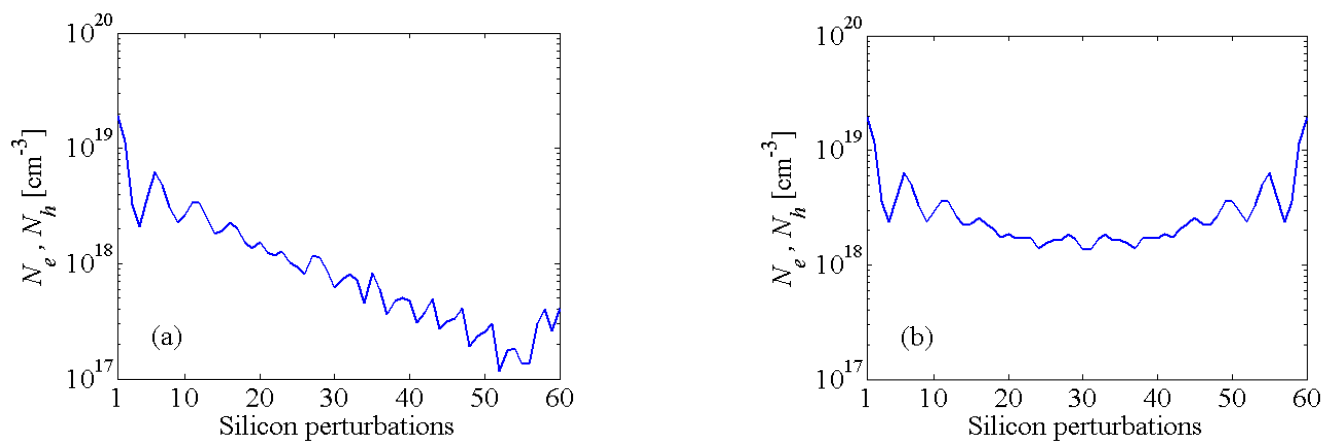

Fig. 2. Profile of carrier concentration versus silicon perturbation cell for (a) single pump and (b) bi-directional pump configuration.

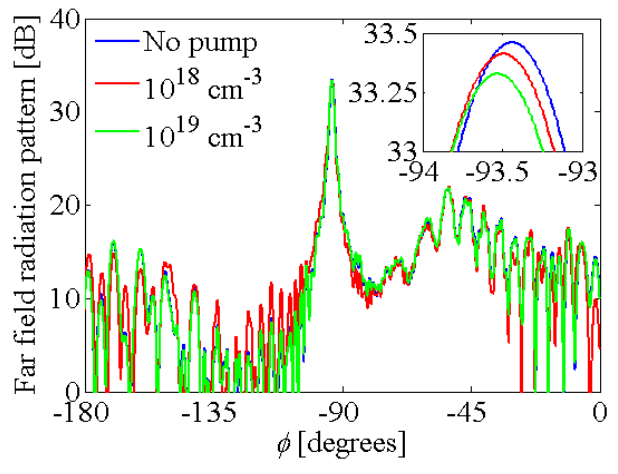

Fig. 3. Comparison of the radiation patterns in the case of no pump, and two values of pump power.

In the bi-directional pumping configuration shown in Fig. 2(b), as a first approximation, the carrier concentration can be considered to be constant in every silicon perturbation. Moreover, the pump power density can be modified to obtain different concentration values. As an example, we suppose to inject enough pumping power to obtain $N_{e}=N_{h}=10^{18} \mathrm{~cm}^{-3}$ and $N_{e}=N_{h}=10^{19} \mathrm{~cm}^{-3}$ (we limit our calculation to this last value since for higher injection levels Auger process becomes dominant and strict calculations should be performed, to be done in future studies); we then observe the radiation patterns at $1550 \mathrm{~nm}$ (Signal waveguide input power density is $4.17 \times 10^{8} \mathrm{~W} / \mathrm{cm}^{2}$ ) in the two mentioned cases, and we compare them to the one in absence of pumping signals in Fig. 3. We observe that, with respect to the case without pump, the beam moves towards more negative angles by about 0.05 and 0.09 degrees for increasing pumping power density to about $2.08 \times 10^{5} \mathrm{~W} / \mathrm{cm}^{2}$ (red curve) and $2.08 \times 10^{6} \mathrm{~W} / \mathrm{cm}^{2}$ (green curve); also, the maximum magnitude of the radiation decreases for increasing pumping power density. Looking at the direction of maximum radiation for the blue curve (around $-93.4^{\circ}$, no pump), the radiation of the red and green curves is about $0.06 \mathrm{~dB}$ and $0.17 \mathrm{~dB}$ lower than the one in absence of the pump.

\section{Conclusion}

We have shown the possibility of controlling the radiation of the analyzed OLWA through optical pumping. The tunability sensitivity will be enhanced in future studies by integrating the OLWA into a resonator.

Acknowledgements: This work is supported by NSF Award \# ECCS-1028727. The authors also thank Ansys, for providing HFSS, and COMSOL Multiphysics.

\section{References}

[1] Q. Song, O. Boyraz and F. Capolino, "Narrow beam radiation from a CMOS compatible leaky wave optical antenna," in Conference on Lasers and Electro-Optics, paper JThE40, San Francisco, CA, 2010.

[2] Q. Song, S. Campione, O. Boyraz and F. Capolino, "Silicon-based optical leaky wave antenna with narrow beam radiation", Opt. Express, 19, 9, 8735-8749 (2011).

[3] O. Boyraz, X. Sang, E.-K. Tien, Q. Song, F. Qian, and M. Akdas, "Silicon based optical pulse shaping and characterization," S. Jiang, M. J. F. Digonnet, J. W. Glesener, and J. C. Dries, eds. (SPIE, San Jose, CA, USA, 2009), pp. 72120U-13. 